\title{
Proving and Generalizing Desargues' Two-Triangle Theorem in 3-Dimensional Projective Space
}

\author{
Dimitrios Kodokostas \\ Department of Applied Mathematics, National Technical University of Athens, Zografou Campus, 15780 Athens, Greece \\ Correspondence should be addressed to Dimitrios Kodokostas; dkodokostas@math.ntua.gr
}

Received 22 August 2014; Accepted 15 November 2014; Published 18 December 2014

Academic Editor: Isaac Pesenson

Copyright ( 2014 Dimitrios Kodokostas. This is an open access article distributed under the Creative Commons Attribution License, which permits unrestricted use, distribution, and reproduction in any medium, provided the original work is properly cited.

With the use of only the incidence axioms we prove and generalize Desargues' two-triangle Theorem in three-dimensional projective space considering an arbitrary number of points on each one of the two distinct planes allowing corresponding points on the two planes to coincide and three points on any of the planes to be collinear. We provide three generalizations and we define the notions of a generalized line and a triangle-connected plane set of points.

\section{The Problem in Perspective}

Perhaps the most important proposition deduced from the axioms of incidence in projective geometry for the projective space is Desargues' two-triangle Theorem usually stated quite concisely as follows:

two triangles in space are perspective from a point if and only if they are perspective from a line

meaning that if we assume a one-to-one correspondence among the vertices of the two triangles, then the lines joining the corresponding vertices are concurrent if and only if the intersections of the lines of the corresponding sides are collinear (Figure 1). According to the ancient Greek mathematician Pappus (3rd century A.D.), this theorem was essentially contained in the lost treatise on Porisms of Euclid (3rd century B.C.) $[1,2]$ but it is nowadays known by the name of the French mathematician and military engineer Gerard Desargues (1593-1662) who published it in 1639. Actually only half of it is called Desargues' Theorem (perspectivity from a point implies perspectivity from a line) whereas the other half is called converse of Desargues' Theorem. All printed copies of Desargues' treatise were lost, but fortunately Desargues' contemporary French mathematician Phillipe de La Hire (1640-1718) made a manuscript copy of it which was discovered again some 200 years later [3].

There exist a few hidden assumptions in the above pretty and compact statement of the theorem. Namely, all mentioned lines and intersection points are assumed to exist and the three points on each plane are assumed to be noncollinear. It seems that in bibliography there exist very few treatments of the theorem paying attention to these assumptions, like Hodge and Pedoe's [4] and Pogorelov's [5]. The purpose of this paper is to deal with these assumptions. It is also worth mentioning that Desargues was interested in triangles in space formed by intersecting two distinct planes with three lines going through a common point. Remarkably, although the theorem remains true even when the two planes of the projective space coincide, it is not at all evident that it holds whenever we work solely in a general projective plane disregarding a larger surrounding projective space. Actually Desargues' Theorem does not hold in the projective geometry of a general projective plane defined by the usual incidence axioms as Hilbert has shown [6], unless if the theorem itself or a statement that implies it is assumed as an axiom. Such a set of axioms was given by Bachmann [7], and a proof of Desargues' Theorem can be found in [8] based on the following Pappus' Theorem of Euclidean Geometry being considered as an axiom:

$$
\begin{aligned}
& \text { if the six vertices of a hexagon lie alternatively on } \\
& \text { two lines, then the three points of intersection of } \\
& \text { pairs of opposite sides are collinear. }
\end{aligned}
$$

The general projective planes came to existence in an attempt to construct via incidence axioms planes in which 


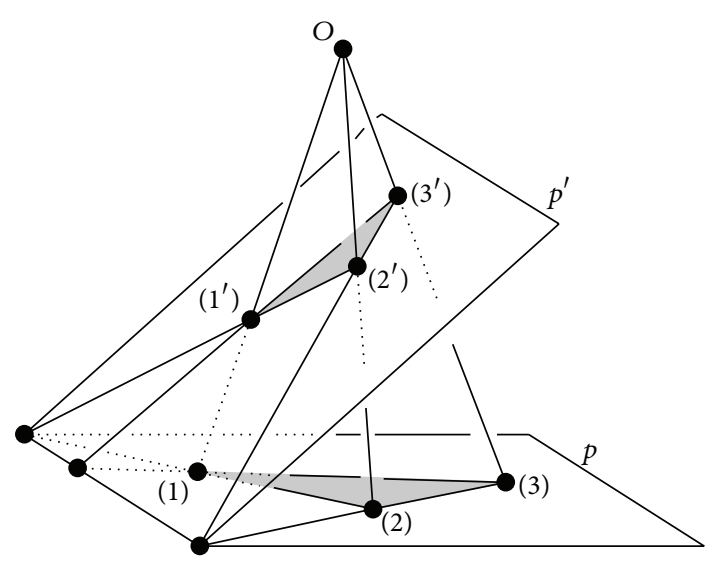

FIGURE 1: Desargues' 2-triangle Theorem in space and its converse.

Desargues' Theorem does or rather does not hold. They are defined as collections of two kinds of objects called points and lines satisfying at least the following three incidence axioms:

(i) any two distinct points are incident with exactly one line;

(ii) any two distinct lines are incident with exactly one point;

(iii) there exist four points of which no three are collinear,

where incidence is used as a neutral word meaning that the point belongs or lies on the line and also meaning that the line passes through or contains the point. Collinearity has the usual meaning. Some projective planes have only finitely many points and lines whereas some others have infinitely many. Desargues' Theorem fails to hold for many of them, both finite and infinite [9-12]. Hilbert [6], Hall [13], and others have proved a plane is Desarguesian (for such planes Desargues' Theorem holds) if and only if it can be constructed algebraically from the vector spaces $K^{3}$ over the division rings $K$, where the points of these planes are just the lines of the vector space $K^{3}$ and the lines of the planes are the subspaces of $K^{3}$ spanned by two linearly independent vectors of it. The usual real projective plane comes by this construction from $\mathbb{R}^{3}$.

Nevertheless, for projective spaces of dimension at least three which are defined similar to the projective planes either by a set of incidence axioms or by algebraic constructions, Desargues' Theorem is always true [4]. These higher dimensional projective spaces contain 2-dimensional projective planes and it is easily seen that Desargues' Theorem holds for all such projective planes too.

We are going to prove and generalize Desargues' Theorem working in a three-dimensional projective space and considering an arbitrary number of points on each one of the two distinct planes, allowing some of the lines and intersection points mentioned in the above statement of the theorem to disappear. For this matter we will allow some of the given points on one of the planes to coincide with their corresponding points on the other plane. Also, although we will always be assuming that the given points are distinct on each plane, we will drop the restriction that any three of them are noncollinear. All our proofs will be based directly on the axioms of projective geometry in space and their immediate corollaries.

The projective space we will be working on consists of three kinds of objects called points, lines, and planes for which the following incidence axioms hold [5]:

(i) there exists exactly one line through any two distinct points;

(ii) any two distinct lines on the same plane have a unique common point;

(iii) there exist at least three points on each line;

(iv) there exist three points not on the same line;

(v) there exist four points not on the same plane;

(vi) there exists exactly one plane through any three distinct noncollinear points; there exists at least one point on each plane;

(vii) if two distinct points are on a plane, then all points in the line containing the given points are on the same plane;

(viii) if two planes have a common point, then they have at least one more common point.

Some immediate corollaries are the following:

(i) a plane and a line not on the plane always have a unique common point;

(ii) two distinct planes intersect in a common line.

A number of given points will be called collinear whenever some line contains all of them (if some points coincide, there may be more than one such line). Similarly, a number of given lines will be called concurrent whenever there exists at least one point lying on all of them. We will say a given set of points on a plane is in general position whenever no three collinear points exist among them. A usual triangle or just a triangle will be a triad of lines defined by three noncollinear points.

\section{A Generalized Desargues' Theorem for Points in General Position Allowing Corresponding Points to Coincide}

Figure 2(a) makes it clear that if we wish to generalize to larger numbers of points the converse of Desargues' Theorem, we have to assume distinct given points on each plane. Similarly, Figure 2(b) says that we should not allow some three collinear points on a plane whenever some of the rest coincide with their corresponding points on the other plane. Nevertheless there do exist generalizations if we allow only one of these two bothersome situations to happen, as we show below.

So let (1), (2), .. be given points on a plane $p$ and $\left(1^{\prime}\right),\left(2^{\prime}\right), \ldots$ some corresponding points on another plane $p^{\prime}$ allowing for the possibility that some corresponding points coincide. Instead of ignoring pairs of coinciding corresponding points we choose to use generalized lines 


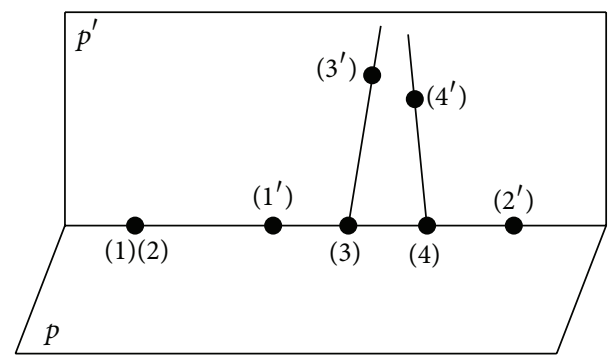

(a)

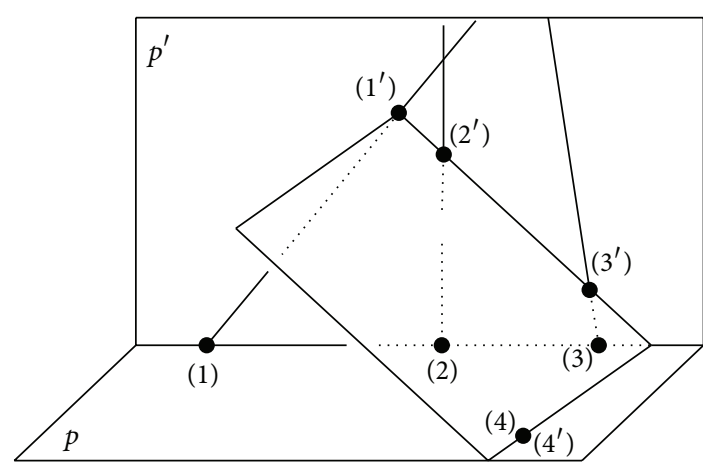

(b)

FIGURE 2: No matter what the meaning of the line (1)(2) in (a) and the line $(4)\left(4^{\prime}\right)$ in (b) is, the lines $(i)\left(i^{\prime}\right)$ cannot all be concurrent.

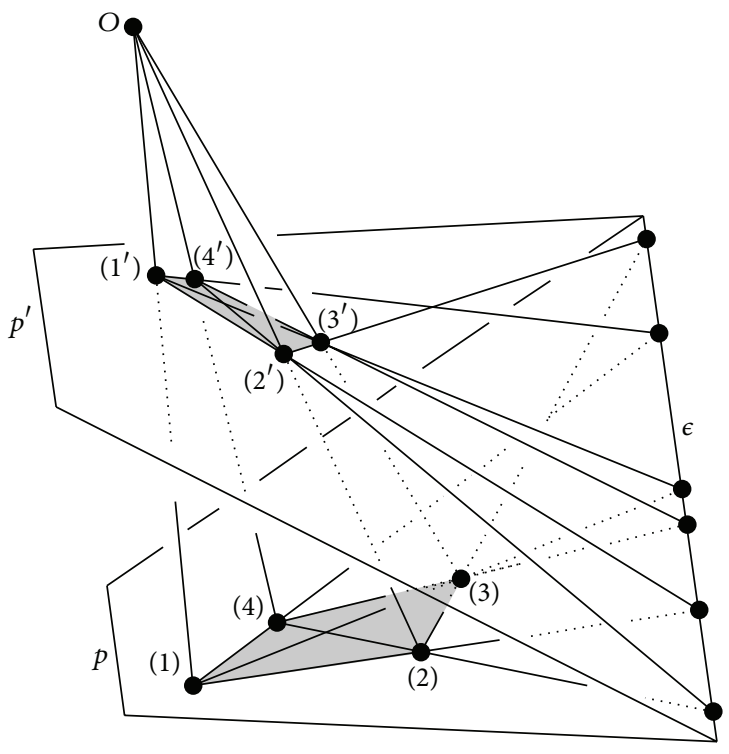

FIGURE 3: Desargues' Theorem for 4 given points on each plane.

defining a generalized line $(i)\left(i^{\prime}\right)$ to be the line through $(i)$ and $\left(i^{\prime}\right)$ whenever $(i),\left(i^{\prime}\right)$ are distinct, otherwise to be the set of all lines through $(i)$ as this seems most natural. It is also convenient to say that $(i)(i)$ contains or goes through all points in our projective space. Our first generalization is the following (Figure 3).

Proposition 1. Let $p, p^{\prime}$ be two distinct planes, (1), (2),..., $(n),(n \in \mathbb{N})$, distinct points on $p$, and $\left(1^{\prime}\right),\left(2^{\prime}\right), \ldots,\left(n^{\prime}\right)$ distinct points on $p^{\prime}$, both sets of points in general position.

(A) If all generalized lines $(i)\left(i^{\prime}\right)$ go through a common point, then all the intersections of the pairs of lines $(i)(j),\left(i^{\prime}\right)\left(j^{\prime}\right)$ for $i \neq j$ are nonempty and lie on a common line (the common line of $p$ and $\left.p^{\prime}\right)$.

(B) If all the intersections of the pairs of lines $(i)(j),\left(i^{\prime}\right)\left(j^{\prime}\right)$ for $i \neq j$ are nonempty, then all generalized lines $(i)\left(i^{\prime}\right)$ go through a common point.
Proof. For $n=1$ part (A) holds vacuously. For $n \geq 2$ note that for arbitrary distinct indices $i, j$ the generalized lines $(i)\left(i^{\prime}\right)$ and $(j)\left(j^{\prime}\right)$ share some common point; thus the lines $(i)(j),\left(i^{\prime}\right)\left(j^{\prime}\right)$ should lie on some common plane (Figure 4 provides a quick pictorial proof) and so they should have a nonempty intersection. But the first of these lines belongs to $p$ whereas the second belongs to $p^{\prime}$ which forces their intersection to lie on $\epsilon$, the common line of the distinct planes $p, p^{\prime}$. Since this happens for all indices $i \neq j$ we are finished with part (A).

The proof of part (B) is a bit longer. For $n=1$ the proposition holds vacuously. For $n=2$ its truth is immediate whenever at least one of the generalized lines $(1)\left(1^{\prime}\right),(2)\left(2^{\prime}\right)$ is not a line, whereas if both of them are lines, they either coincide (both with the common line of $p$ and $p^{\prime}$ ) and the results hold trivially, or else they are distinct, in which case the four points (1), ( $\left.1^{\prime}\right),(2),\left(2^{\prime}\right)$ do not lie on the same line. But then the lines $(1)(2),\left(1^{\prime}\right)\left(2^{\prime}\right)$ are also distinct, and as their intersection is nonempty by assumption, they define a plane on which lie the points $(1),\left(1^{\prime}\right),(2),\left(2^{\prime}\right)$ forcing the lines $(1)\left(1^{\prime}\right),(2)\left(2^{\prime}\right)$ to lie on this plane too and thus to have nonempty intersection (a single point, since these lines do not coincide).

For $n=3$ and assuming that at least one of the generalized lines $(1)\left(1^{\prime}\right),(2)\left(2^{\prime}\right),(3)\left(3^{\prime}\right)$ is not a line, say $(3)\left(3^{\prime}\right)$, the result comes from the truth of case $n=2$, since $(1)\left(1^{\prime}\right),(2)\left(2^{\prime}\right)$ have to share some common point which of course lies on the generalized line (3) $\left(3^{\prime}\right)$ as well. So assume that all three of these generalized lines are lines (Figure 1), which is the classical assumption for the converse of Desargues' Theorem.

Observe that at least one of the points $\left(1^{\prime}\right),\left(2^{\prime}\right),\left(3^{\prime}\right)$, say $\left(1^{\prime}\right)$, does not lie on $p$; otherwise the plane $p^{\prime}$ defined by these three noncollinear points would coincide with $p$, which cannot happen. Then the line $\left(1^{\prime}\right)\left(2^{\prime}\right)$ does not lie on $p$ either, and so it is distinct from the line (1)(2) which lies on $p$, and since $\left(1^{\prime}\right)\left(2^{\prime}\right),(1)\left(2^{\prime}\right)$ have a nonempty intersection, they define a plane, say $p_{12}$. The line (3) $\left(3^{\prime}\right)$ cannot lie on $p_{12}$ for if it did, the three noncollinear points (1), (2), (3) would lie on $p_{12}$ making it coincide with $p$, and then point $\left(1^{\prime}\right)$ of $p_{12}$ would be a point of $p$, a contradiction. So the line (3) $\left(3^{\prime}\right)$ has a unique common point $O$ with $p_{12}$. And we show below that $(3)\left(3^{\prime}\right)$ has a unique intersection point with either of lines 


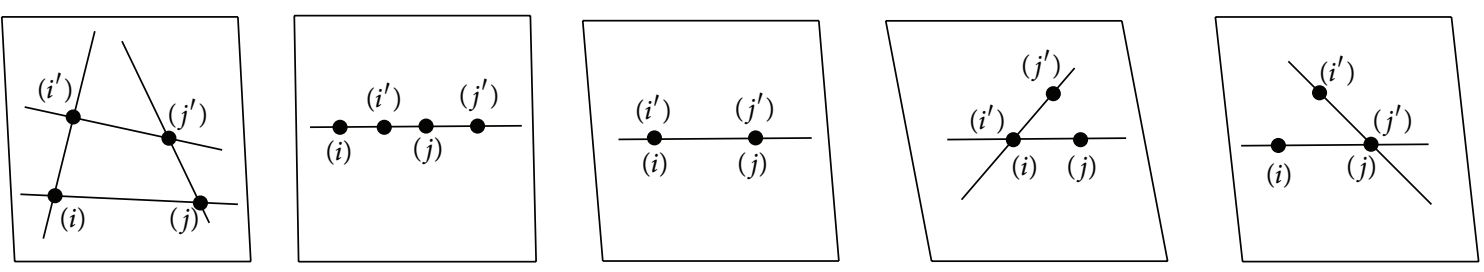

FIGURE 4: Whenever the generalized lines $(i)\left(i^{\prime}\right),(j)\left(j^{\prime}\right)$ share a common point, the lines $(i)(j),\left(i^{\prime}\right)\left(j^{\prime}\right)$ lie on some common plane.

$(1)\left(1^{\prime}\right),(2)\left(2^{\prime}\right)$ of $p_{12}$. Therefore $(1)\left(1^{\prime}\right),(2)\left(2^{\prime}\right),(3)\left(3^{\prime}\right)$ have to go through a common point $O$ as wanted. Now for the claim about the intersections of $(3)\left(3^{\prime}\right)$ with $(1)\left(1^{\prime}\right),\left(2^{\prime}\right)\left(2^{\prime}\right)$, the lines $(1)(3),\left(1^{\prime}\right)\left(3^{\prime}\right)$ are distinct; otherwise the lines $(1)\left(1^{\prime}\right),(3)\left(3^{\prime}\right)$ would coincide forcing $(3)\left(3^{\prime}\right)$ to lie on $p_{12}$ which it cannot do. Then since by hypothesis $(1)(3),\left(1^{\prime}\right)\left(3^{\prime}\right)$ intersect nonvacuously they define a plane on which all four points $(1),(3),\left(1^{\prime}\right),\left(3^{\prime}\right)$ lie, making the lines $(1)\left(1^{\prime}\right),(3)\left(3^{\prime}\right)$ lie on it and so have a nonempty intersection. This intersection is actually a unique point; otherwise the lines $(1)\left(1^{\prime}\right),(3)\left(3^{\prime}\right)$ would coincide forcing $(3)\left(3^{\prime}\right)$ to lie on $p_{12}$, a contradiction. Quite similarly $(3)\left(3^{\prime}\right)$ intersects $\left(2^{\prime}\right)\left(2^{\prime}\right)$ in a unique point as wanted.

For $n \geq 4$ there exist at least $n-2 \geq 2$ lines among the (i) $\left(i^{\prime}\right)$ 's for, if not, at least three (i)'s would coincide with the corresponding $\left(i^{\prime}\right)^{\prime}$ 's, and all these $(i)$ would be collinear lying on the common line of $p$ and $p^{\prime}$ contrary to the proposition's assumption.

If there exist exactly two lines among the $(i)\left(i^{\prime}\right)^{\prime}$ 's (which can happen only for $n=4)$, say $(1)\left(1^{\prime}\right)$ and $(2)\left(2^{\prime}\right)$, then for $i>2$ the points $(i)$ and $\left(i^{\prime}\right)$ coincide and lie on the common line $\epsilon$ of $p, p^{\prime}$. Now the lines (1)(2), $\left(1^{\prime}\right)\left(2^{\prime}\right)$ cannot coincide; otherwise they would lie on both $p, p^{\prime}$, and so they would coincide with $\epsilon$; thus all points (i) would lie on $\epsilon$, contrary to our original assumption. Since $(1)(2),\left(1^{\prime}\right)\left(2^{\prime}\right)$ are distinct and by assumption intersect nonvacuously, they define a plane on which lie all four points $(1),\left(1^{\prime}\right),(2),\left(2^{\prime}\right)$. So both lines $(1)\left(1^{\prime}\right),(2)\left(2^{\prime}\right)$ lie on this plane as well, and they must have a nonempty intersection, a single point. Of course all generalized lines $(i)\left(i^{\prime}\right)$ go through this point as well.

Finally, if there exist at least three lines among the $(i)\left(i^{\prime}\right)^{\prime}$ 's, say $(1)\left(1^{\prime}\right), \ldots,(k)\left(k^{\prime}\right)$ with $k \geq 3$, then according to the proof of case $n=3$, for any $j, 3 \leq j \leq k$, the lines $(1)\left(1^{\prime}\right),(2)\left(2^{\prime}\right)$, and $(j)\left(j^{\prime}\right)$ are distinct and intersect each other in a single point which makes all three of them go through a common point, say $O_{j}$. Since $O_{j}$ is the unique common point of the distinct lines (see scholium below) $(1)\left(1^{\prime}\right),(2)\left(2^{\prime}\right)$ for all indices $j$, all these points coincide and the lines $(1)\left(1^{\prime}\right), \ldots,(k)\left(k^{\prime}\right)$ go through this common point, which of course is also a point of any generalized $(i)\left(i^{\prime}\right)$ as wanted, finishing the proof of the proposition.

Remark 2. The above proof reveals that with the classical hypothesis for the converse of Desargues' Theorem the three lines $(1)\left(1^{\prime}\right),(2)\left(2^{\prime}\right),(3)\left(3^{\prime}\right)$ are distinct and the three planes defined by two of them at a time are distinct, a result to be used in the next section. Indeed, with the assumptions made in the proof, line $(3)\left(3^{\prime}\right)$ is distinct from the other two since

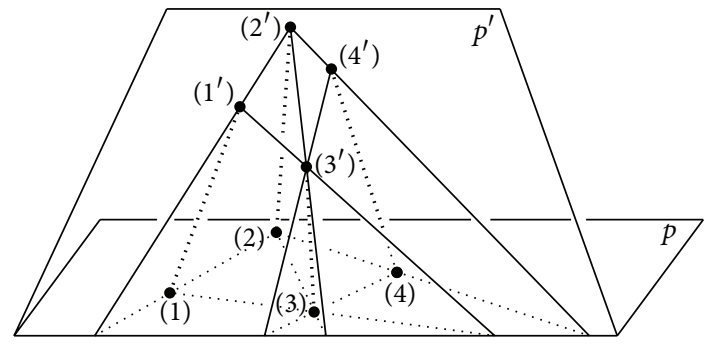

FiguRE 5: If $(1)\left(1^{\prime}\right),(2)\left(2^{\prime}\right),(3)\left(3^{\prime}\right)$ are concurrent and $(2)\left(2^{\prime}\right)$, $(3)\left(3^{\prime}\right),(4)\left(4^{\prime}\right)$ are concurrent, then all $(1)\left(1^{\prime}\right),(2)\left(2^{\prime}\right),(3)\left(3^{\prime}\right)$, (4) $\left(4^{\prime}\right)$ are concurrent.

it does not lie on the plane $p_{12}$ defined by them, whereas if $(1)\left(1^{\prime}\right),(2)\left(2^{\prime}\right)$ were not distinct, the points $(1),\left(1^{\prime}\right),(2),\left(2^{\prime}\right)$ would lie on the same line and this line would be of course (1)(2), forcing $\left(1^{\prime}\right)$ to lie on (1)(2) and therefore on $p$, a contradiction. And any two of the planes defined by two of $(1)\left(1^{\prime}\right),(2)\left(2^{\prime}\right),(3)\left(3^{\prime}\right)$ have to be distinct since otherwise all points $(1),(2),(3),\left(1^{\prime}\right),\left(2^{\prime}\right),\left(3^{\prime}\right)$ would lie on the same plane forcing $p$ and $p^{\prime}$ to coincide, which cannot be.

\section{A Generalization for the Converse of Desargues' Theorem for Triangle-Connected Given Points}

The assumptions postulated in part (B) of Proposition 1 can be considerably relaxed. For example, there is no reason for assuming that all lines $(i)(j)$ intersect with their corresponding lines $\left(i^{\prime}\right)\left(j^{\prime}\right)$, since a much smaller number of lines among the $(i)(j)$ 's with the same property force all the rest to behave similarly.

This is made clear in Figure 5 where the lines of the sides of triangle (1)(2)(3) intersect their corresponding lines of triangle $\left(1^{\prime}\right)\left(2^{\prime}\right)\left(3^{\prime}\right)$ making the lines $(1)\left(1^{\prime}\right),(2)\left(2^{\prime}\right),(3)\left(3^{\prime}\right)$ go through the same point $O_{1}$, and similarly the lines of the sides of triangle (2)(3)(4) intersect their corresponding lines of triangle $\left(2^{\prime}\right)\left(3^{\prime}\right)\left(4^{\prime}\right)$ making the lines $(2)\left(2^{\prime}\right),(3)\left(3^{\prime}\right),(4)\left(4^{\prime}\right)$ go through the same point $\mathrm{O}_{2}$. Since both $\mathrm{O}_{1}, \mathrm{O}_{2}$ coincide with the intersection point of $(1)\left(1^{\prime}\right)$ and $(2)\left(2^{\prime}\right)$ they have to coincide with each other, thus making all lines $(1)\left(1^{\prime}\right),(2)\left(2^{\prime}\right),(3)\left(3^{\prime}\right),(4)\left(4^{\prime}\right)$ go through a common point. Then by part (A) of Proposition 1, lines $(1)(4),\left(1^{\prime}\right)\left(4^{\prime}\right)$ have to intersect as well. Of course all the intersections of the pairs of lines $(i)(j),\left(i^{\prime}\right)\left(j^{\prime}\right)$ have to lie on the common line of $p$ and $p^{\prime}$. 


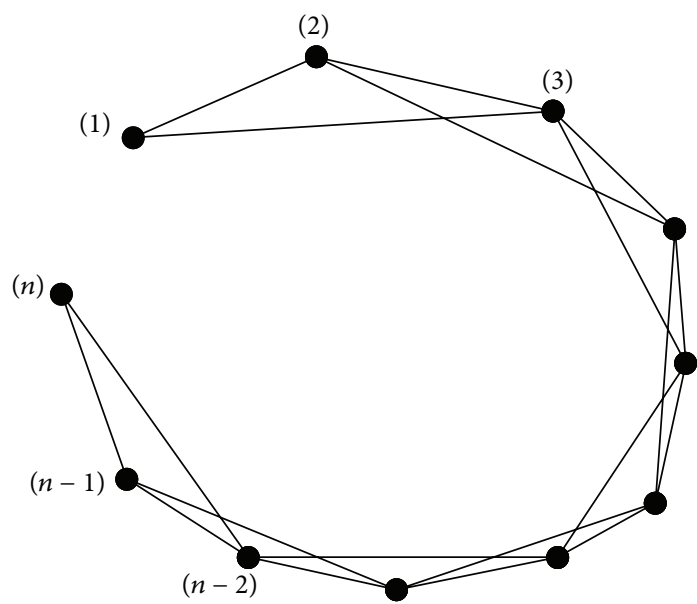

(a)

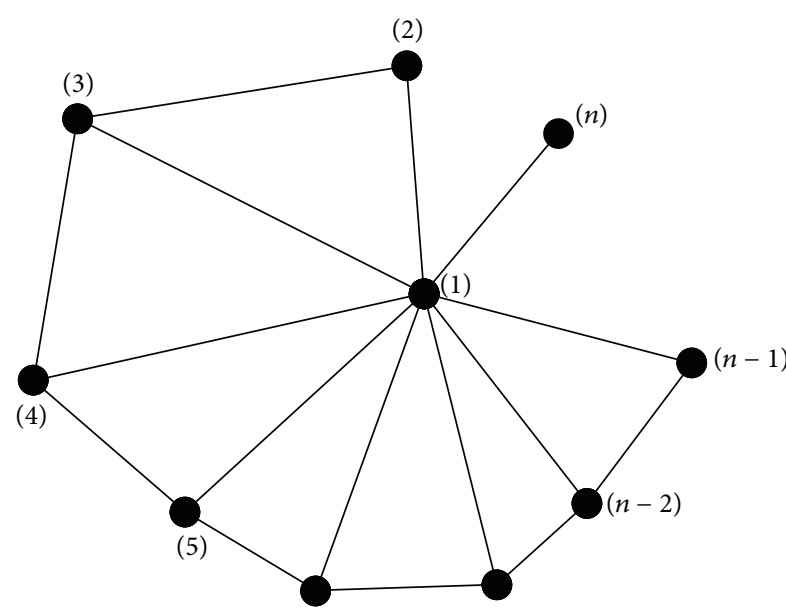

(b)

Figure 6: (a) A triangle-connection set for the points (1), (2), ., , (n). (b) $n-4$ in number lines can never be all the triangle-connection lines for $n$ points, no three of which are collinear.

Also note that the points (1), (2), (3), and (4) need not be noncollinear by three; in the argument above we only needed the existence of the triangles (1)(2)(3) and (2)(3)(4) and their corresponding ones in $p^{\prime}$. Finally note that if we expand the given sets of points with some more points $(5)=\left(5^{\prime}\right),(6)=$ $\left(6^{\prime}\right), \ldots$ all in the common line of $p$ and $p^{\prime}$, then trivially all lines $(1)\left(1^{\prime}\right),(2)\left(2^{\prime}\right), \ldots$ continue to share a common point.

This argument extends similarly for any $n \geq 3$ given points $(i)$ and $\left(i^{\prime}\right)$ on the two planes. All we need is to assume that all points $(i)$ that do not coincide with their corresponding points $\left(i^{\prime}\right)$ are similarly triangle-connected on the two planes. The meaning of this should be rather clear.

A triangle-connection sequence in $S$ or for simplicity just triangle-connection sequence for two points $A, B$ of a given set $S$ will be any finite sequence of triangles formed by lines through the points of $S$ so that any two consecutive triangles share a common side and $A$ belongs to the first triangle of this sequence whereas $B$ belongs to the last one; recall from Section 1 that a triangle is a set of three lines. A triangleconnection set for $S$ will be a set of triangle-connection sequences of points of $S$, that contains exactly one such sequence for each pair of points of $S$. Of course whenever such a set exists for $S$, it is not necessarily unique. The triangles in the sequences of such a set will be called connection triangles for $S$ and the lines of the sides connection lines. Two given sets of points $(1), \ldots,(n)$ and $\left(1^{\prime}\right), \ldots,\left(n^{\prime}\right)$ on two planes $p, p^{\prime}$ where $(i)$ corresponds to $\left(i^{\prime}\right)$ will be called similarly triangleconnected whenever there exists a triangle-connection set for the $(i)$ 's which becomes a triangle-connection set for the $\left(i^{\prime}\right)$ 's by replacing all vertices of all triangles in the sequences of this set by their corresponding points on the other plane.

For example, a triangle-connection set for the set $S=$ $\{(1),(2), \ldots,(n)\}$ in Figure 6(a) is $\left\{S_{i j} \mid i \leq j+2\right\}$, where $S_{i j}=((i)(i+1)(i+2),(i+1)(i+2)(i+3), \ldots,(j-2)(j-1)(j))$ and triangles are denoted by the three names of their vertices. The triangle-connection lines in this set are (1)(2), (2)(3), .., $(n-$ $1)(n)$ and $(1)(3),(2)(4), \ldots,(n-2)(n)$, that is, $2 n-3$ lines in total. It is interesting to note by the way that the minimum number of connection lines in a triangle-connection set of lines for $n$ given points no three of which are collinear is $2 n-3$. Figure 6(b) hints at the reason; a rigorous proof is easy to come by.

Now for two similarly triangle-connected sets of points (i) and $\left(i^{\prime}\right)$ on two planes $p$ and $p^{\prime}$ and assuming (1)(2)(3) to be a connection triangle on one of the planes, we know by the remark after Proposition 1 that the lines $(1)\left(1^{\prime}\right),(2)\left(2^{\prime}\right),(3)\left(3^{\prime}\right)$ are distinct and that all three intersect in a single point $O$. It is quite trivial to show, and we do so in the next paragraph, that every line $(i)\left(i^{\prime}\right)$ intersects all three lines $(1)\left(1^{\prime}\right),(2)\left(2^{\prime}\right),(3)\left(3^{\prime}\right)$ nonvacuously for all $i$. Since, for $i \neq 1$, the line $(i)\left(i^{\prime}\right)$ does not belong to at least one of the distinct planes defined by $(1)\left(1^{\prime}\right),(2)\left(2^{\prime}\right)$ and $(1)\left(1^{\prime}\right),(3)\left(3^{\prime}\right)$, we get that $(i)\left(i^{\prime}\right)$ has a unique point with at least one of the planes. In other words $(i)\left(i^{\prime}\right)$ either intersects $(1)\left(1^{\prime}\right)$ and (2) $\left(2^{\prime}\right)$ of the first of these planes on their common point $O$ or else intersects $(1)\left(1^{\prime}\right)$ and $(3)\left(3^{\prime}\right)$ of the second plane on their common point $O$. So all lines $(i)\left(i^{\prime}\right)$ go through $O$ proving Proposition 3 below, which is a generalization of the converse of Desargues' Theorem.

About the claim that for all $i$ the line $(i)\left(i^{\prime}\right)$ intersects nonvacuously all three lines $(1)\left(1^{\prime}\right),(2)\left(2^{\prime}\right),(3)\left(3^{\prime}\right)$, for $(i)=$ $\left(i^{\prime}\right)$ this is immediate, so consider an $i$ so that $(i)\left(i^{\prime}\right)$ is a usual line and choose a triangle-connection sequence for (1) and $(i)$. For any triangle $\left(i_{1}\right)\left(i_{2}\right)\left(i_{3}\right)$ in this sequence and its corresponding $\left(i_{1}^{\prime}\right)\left(i_{2}^{\prime}\right)\left(i_{3}^{\prime}\right)$ on the other plane, the assumptions for the converse of the classical Desargues Theorem are satisfied so (see the scholium) the lines $\left(i_{1}\right)\left(i_{1}^{\prime}\right),\left(i_{2}\right)\left(i_{2}^{\prime}\right),\left(i_{3}\right)\left(i_{3}^{\prime}\right)$ are distinct and all three intersect in a single point. Now for two such consecutive triangles $\left(i_{1}\right)\left(i_{2}\right)\left(i_{3}\right),\left(i_{2}\right)\left(i_{3}\right)\left(i_{4}\right)$ the two concurrency points are the same since they both coincide with the unique intersection point of $\left(i_{2}\right)\left(i_{2}^{\prime}\right)$ and $\left(i_{3}\right)\left(i_{3}^{\prime}\right)$. This implies that all lines $(j)\left(j^{\prime}\right)$ for all the vertices $(j),\left(j^{\prime}\right)$ of all triangles in these triangle-connection sequences have to go through a common point, which implies $(1)\left(1^{\prime}\right)$ and $(i)\left(i^{\prime}\right)$ 


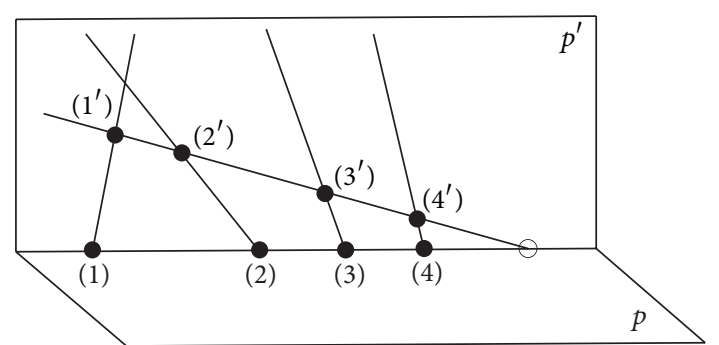

FIgure 7: The converse of Desargues' Theorem does not always hold in case all given points are collinear in both planes.

have some common point. Similarly, $(i)\left(i^{\prime}\right)$ intersects $(2)\left(2^{\prime}\right)$ and $(3)\left(3^{\prime}\right)$ as well and we are done.

Proposition 3. Let $p, p^{\prime}$ be two distinct planes, (1), (2), ..., (n) distinct points on $p$, and $\left(1^{\prime}\right),\left(2^{\prime}\right), \ldots,\left(n^{\prime}\right)$ distinct points on $p^{\prime}$ where $(i),\left(i^{\prime}\right)$ are considered to be corresponding. Assume that for the given points on the two planes which do not coincide with their corresponding points there exist similar triangleconnection sets so that every pair of corresponding triangleconnection lines $(i)(j),\left(i^{\prime}\right)\left(j^{\prime}\right)$ has a nonempty intersection. Then all generalized lines $(i)\left(i^{\prime}\right)$ go through a common point. Moreover, all pairs of lines $(i)(j),\left(i^{\prime}\right)\left(j^{\prime}\right)$ for $i \neq j$ intersect nonvacuously (on the common line of $p$ and $p^{\prime}$ ).

The existence of the triangle-connection sets guarantees that $n \geq 3$ and that at least three points on each of the planes do not coincide with their corresponding points on the other. If exactly two points on each plane do not coincide with their corresponding points, then all but two lines $(i)(i)$ are generalized but the remaining two are not guaranteed to have a nonempty intersection and thus this case cannot be included to the proposition.

\section{Whenever Three or More Collinear Points Are Allowed but Corresponding Points Are Assumed Distinct}

We close with a third generalization of the converse of Desargues' Theorem. In Proposition 1 the given points on the two planes were allowed to coincide with their corresponding points, but no three given points on any of the planes were allowed to be collinear. Flipping over these assumptions and restrictions, we now forbid any two corresponding points to coincide but allow three or more of them on any of the planes to be collinear. Figure 7 reveals that the converse of Desargues' Theorem does not always hold then; at least it does not hold whenever the given points on the planes are all collinear. Interestingly enough, it does hold whenever the given points on one of the planes do not all lie in a single line.

Proposition 4. Let $p, p^{\prime}$ be two distinct planes, (1), (2),..., $(n), n \geq 3$, distinct points on $p$, and $\left(1^{\prime}\right),\left(2^{\prime}\right), \ldots,\left(n^{\prime}\right)$ distinct points on $p^{\prime}$ so that $(i) \neq\left(i^{\prime}\right)$ for all $i$. If all intersections $(i)(j) \cap\left(i^{\prime}\right)\left(j^{\prime}\right)$ are nonempty and not all of the given points are collinear on at least one of the given planes, then all lines (i) $\left(i^{\prime}\right)$ go through a common point.

Proof. For $n=3$ this is exactly part (B) of Proposition 1 . Similarly, it is part (B) of Proposition 1 whenever $n=4$ and no three collinear points exist among the given ones in any of the planes. For $n=4$ and assuming (1), (2), (3) to be collinear on $p$, then (4) does not lie on the line of the other three and so (1)(2)(4), (2)(4)(3) are usual nontrivial triangles, the points (1), (2), (3), (4) are triangle-connected, and our proposition holds because of Proposition 3.

Finally, for $n \geq 5$, we proceed inductively assuming the result holds for all $k \leq n$ : by the proposition's assumptions we can consider $(i)$ to be not all collinear. If all but one of them are collinear, say (1) not on the line of the rest, then $(1)(2)(3),(1)(3)(4), \ldots,(1)(n-1)(1)$ form a triangleconnection set for the $(i)$ 's and the result follows from Proposition 3.

If on the other hand no more than $n-2$ of the (i)'s are collinear, choose two of them, say (1), (2), not on the common line $\epsilon$ of $p, p^{\prime}$. Now observe that the two sets of $n-1$ in number points (1), (2), .., $(n-2),(n-1)$ and (1), (2), .., (n$2),(n)$ satisfy the assumptions of the proposition; thus by our induction hypothesis the result holds for them. In other words, all lines $(2)\left(2^{\prime}\right),(3)\left(3^{\prime}\right), \ldots,(n-2)\left(n-2^{\prime}\right),(n-1)(n-$ $\left.1^{\prime}\right)$ go through a common point $O_{1}$ of $(1)\left(1^{\prime}\right),(2)\left(2^{\prime}\right)$ and similarly all lines $(2)\left(2^{\prime}\right),(3)\left(3^{\prime}\right), \ldots,(n-2)\left(n-2^{\prime}\right),(n)\left(n^{\prime}\right)$ go through a point $O_{2}$ of $(1)\left(1^{\prime}\right),(2)\left(2^{\prime}\right)$. But points $O_{1}, O_{2}$ coincide because the lines $(1)\left(1^{\prime}\right),(2)\left(2^{\prime}\right)$ are distinct: if not, $(1)\left(1^{\prime}\right),(2)\left(2^{\prime}\right)$ would coincide with $\epsilon$ because they would be the line (1)(2) of $p$ and at the same time the line $\left(1^{\prime}\right)\left(2^{\prime}\right)$ of $p^{\prime}$; this would make (1), (2) points of $\epsilon$, a contradiction. So all lines $(i)\left(i^{\prime}\right)$ go through $O_{1}$ and we are done.

\section{Conflict of Interests}

The author declares that there is no conflict of interests regarding the publication of this paper.

\section{References}

[1] H. Dörrie, 100 Great Problems of Elementary Mathematics: Their History and Solution, Dover, 1965.

[2] W. R. Knorr, The Ancient Tradition of Geometric Problems, Dover Publications, New York, NY, USA, 1993.

[3] M. Kline, Mathematics in Western Culture, Oxford University Press, 1967.

[4] W. V. Hodge and D. Pedoe, Methods of Algebraic Geometry, vol. 1, Cambridge University Press, Cambridge, UK, 1953.

[5] A. Pogorelov, Geometry, Mir Publishers, Moscow, Russia, 1987.

[6] D. Hilbert, Grundlagen der Geometrie, 1899, (translated as The Foundations of Geometry and first published in English by The Open Court Publishing Company, Chicago, 1992 and offered as an ebook by Project Gutenberg, 2005, https://www.gutenberg.org/).

[7] I. Bachmann, Aufbau der Geometrie aus dem Spiegelungsbegriff, Grundlehren der Mathematischen Wissenschaften 96, Springer, Berlin, Germany, 2nd edition, 1973.

[8] H. S. M. Coxeter, Introduction to Geometry, John Wiley \& Sons, Inc, New York, NY, USA, 2nd edition, 1989. 
[9] K. Levenberg, "A class of non-desarguesian plane geometries," The American Mathematical Monthly, vol. 57, pp. 381-387, 1950.

[10] F. Moulton, "A simple non-Desarguesian plane geometry," Transactions of the American Mathematical Society, vol. 3, pp. 102-195, 1901.

[11] K. Sitaram, "A real non-desarguesian plane," The American Mathematical Monthly, vol. 70, pp. 522-525, 1963.

[12] O. Veblen and J. H. Maclagan-Wedderburn, "Non-Desarguesian and non-Pascalian geometries," Transactions of the American Mathematical Society, vol. 8, no. 3, pp. 379-388, 1907.

[13] M. Hall, "Projective planes," Transactions of the American Mathematical Society, vol. 54, pp. 229-277, 1943. 


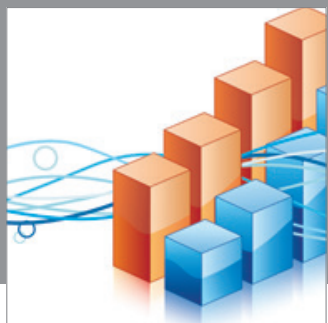

Advances in

Operations Research

mansans

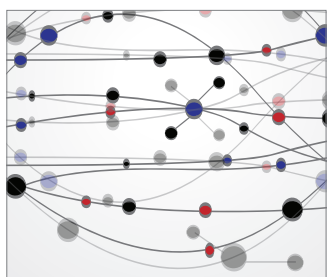

The Scientific World Journal
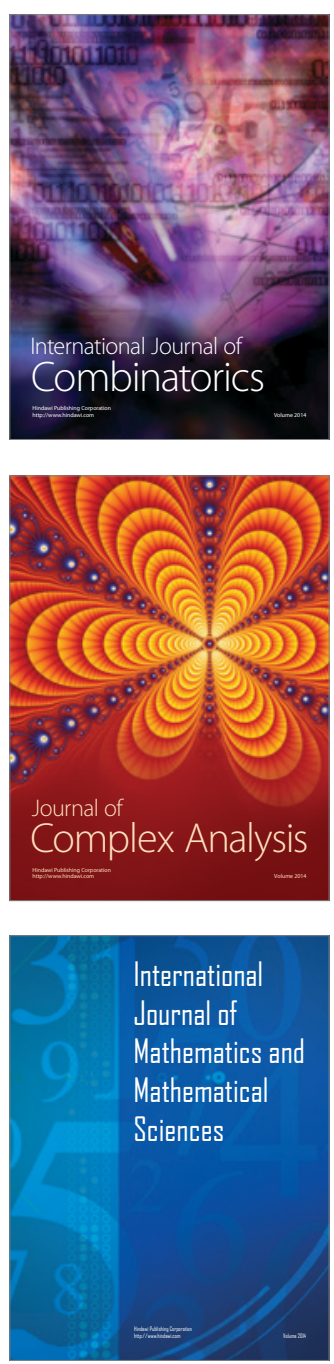
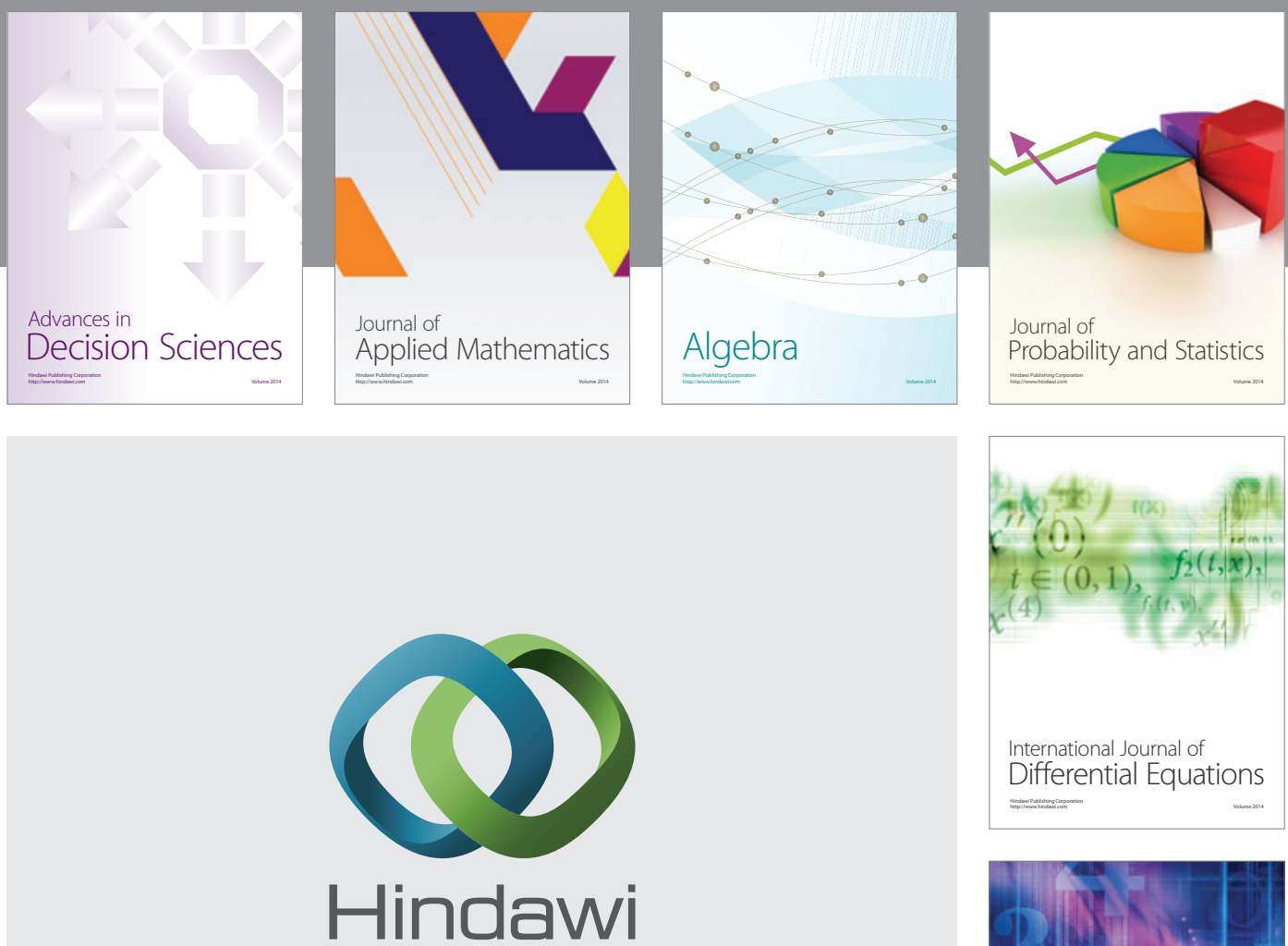

Submit your manuscripts at http://www.hindawi.com
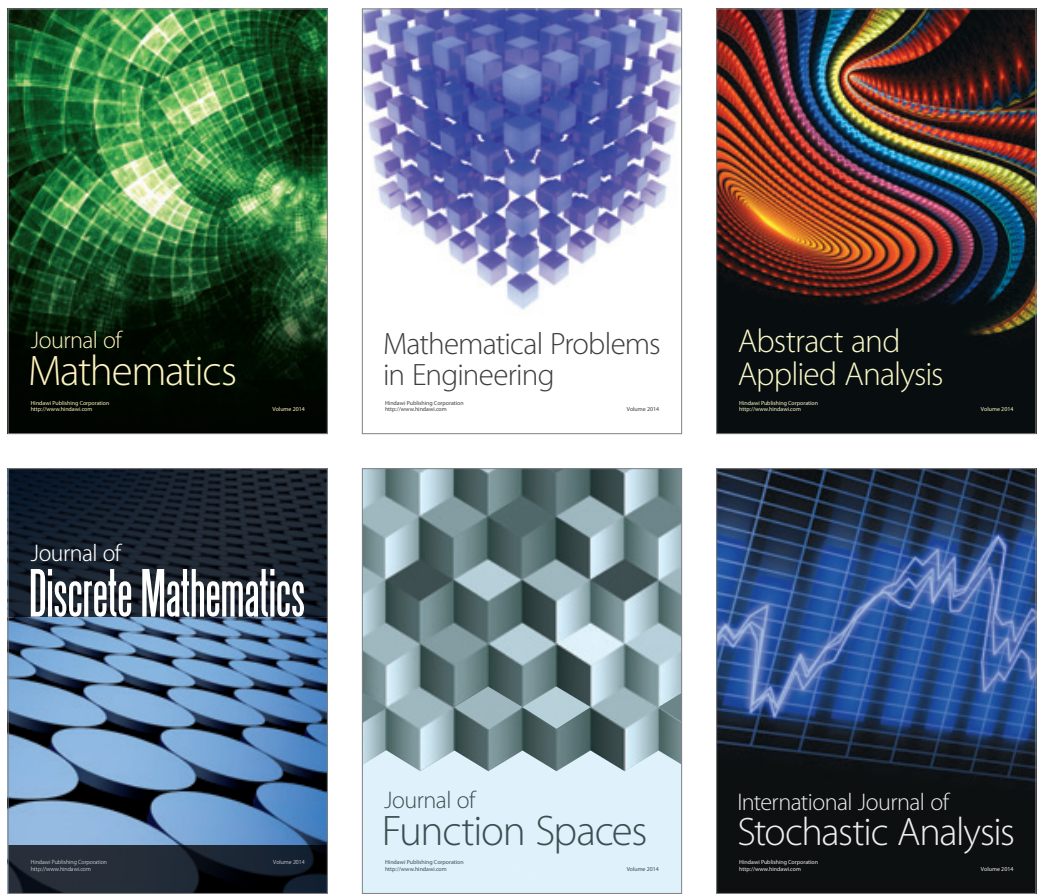

Journal of

Function Spaces

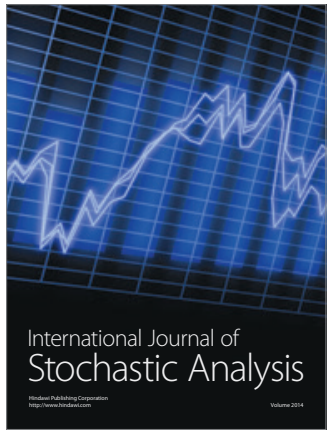

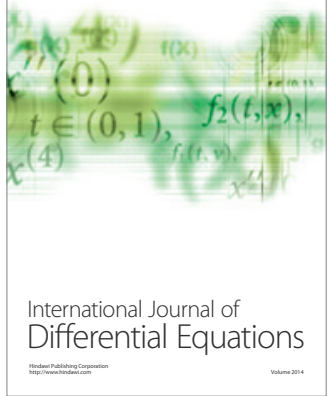
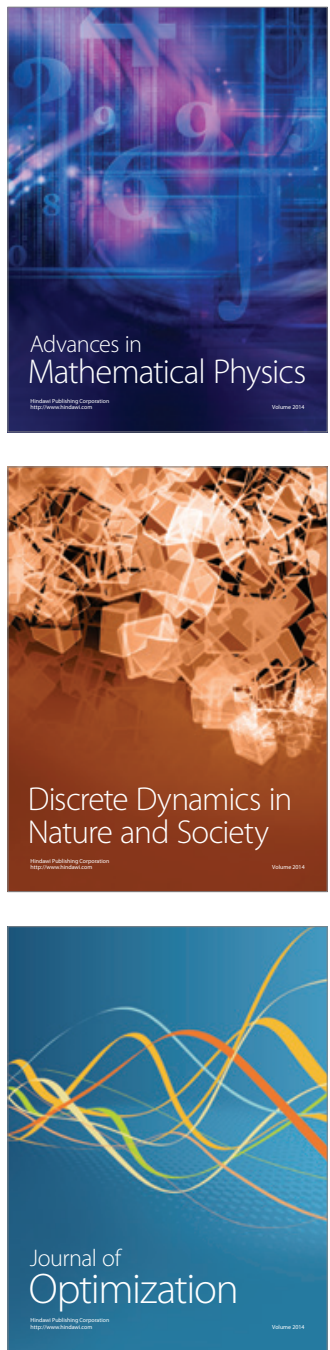